\title{
TITLE:
}

\section{Degenerate fermi gases of ytterbium}

AUTHOR(S):

Fukuhara, T; Takasu, Y; Kumakura, M; Takahashi, Y

CITATION:

Fukuhara, $T$...[et al]. Degenerate fermi gases of ytterbium. PHYSICAL REVIEW LETTERS 2007, 98(3): 030401.

ISSUE DATE:

2007-01-19

URL:

http://hdl.handle.net/2433/50043

RIGHT:

Copyright 2007 American Physical Society 


\title{
Degenerate Fermi Gases of Ytterbium
}

\author{
Takeshi Fukuhara, ${ }^{1}$ Yosuke Takasu, ${ }^{2}$ Mitsutaka Kumakura, ${ }^{1,3,4}$ and Yoshiro Takahashi ${ }^{1,3}$ \\ ${ }^{1}$ Department of Physics, Graduate School of Science, Kyoto University, Kyoto 606-8502, Japan \\ ${ }^{2}$ Department of Electronic Science and Engineering, Graduate School of Engineering, Kyoto University, Kyoto 615-8510, Japan \\ ${ }^{3}$ CREST, Japan Science and Technology Agency, Kawaguchi, Saitama 332-0012, Japan \\ ${ }^{4}$ PRESTO, Japan Science and Technology Agency, Kawaguchi, Saitama 332-0012, Japan
}

(Received 8 July 2006; published 17 January 2007)

\begin{abstract}
Evaporative cooling was performed to cool fermionic ${ }^{173} \mathrm{Yb}$ atoms in a crossed optical dipole trap. The large elastic collision rate leads to efficient evaporation and we have successfully cooled the atoms to $0.37 \pm 0.06$ of the Fermi temperature, that is to say, to a quantum degenerate regime. In this regime, a plunge of evaporation efficiency was observed as a result of Fermi degeneracy.
\end{abstract}

DOI: $10.1103 /$ PhysRevLett.98.030401

PACS numbers: 03.75.Ss, 32.80.Pj, 34.50.-s

Ultracold fermionic gases have been extensively studied in recent years, since the first realization of degenerate Fermi gases of ${ }^{40} \mathrm{~K}$ [1]. Degenerate ${ }^{40} \mathrm{~K}$ atoms were also obtained by sympathetic cooling with ${ }^{87} \mathrm{Rb}$ atoms [2]. A gas of ${ }^{6} \mathrm{Li}$ atoms was cooled to degeneracy by sympathetic cooling with bosonic ${ }^{7} \mathrm{Li} \mathrm{[3,4],}{ }^{23} \mathrm{Na}[5]$, and ${ }^{87} \mathrm{Rb}[6]$, and also in a two-spin state mixture in an optical dipole trap [7]. These degenerate atom clouds have been applied to the investigation of the crossover between Bardeen-CooperSchrieffer superfluidity and Bose-Einstein condensation (BEC) of molecules, and these investigations have yielded significant progress in our understanding of such phenomena [8]. In systems of degenerate Fermi gases in an optical lattice, a variety of interesting achievements, for example, fermionic Bloch oscillations [9] and observation of Fermi surfaces [10], is reported. So far, Fermi degeneracy has been realized only in the three atom species ${ }^{6} \mathrm{Li}$, ${ }^{40} \mathrm{~K}$, metastable ${ }^{3} \mathrm{He}$ [11]. It is quite likely that degenerate Fermi gases of other atoms would have properties that differ greatly from those of alkali and metastable ${ }^{3} \mathrm{He}$ atoms, and thus their production could create new possibilities in the study of ultracold Fermi gases.

Rare-earth atoms of ytterbium ( $\mathrm{Yb}$ ) have a unique advantage in studying Fermi gases because there exist two stable fermionic isotopes, whose natural abundance is more than ten percent: ${ }^{171} \mathrm{Yb}(14.3 \%)$ and ${ }^{173} \mathrm{Yb}(16.1 \%)$ with nuclear spin $I=\frac{1}{2}$ and $I=\frac{5}{2}$, respectively. By using these two isotopes, we can do experiments on Fermi-Fermi mixtures. There are also five bosonic isotopes, which provide us with various possibilities for a sympathetic cooling pair with favorable collision properties to realize degenerate Bose-Fermi mixtures. Furthermore, unique features coming from the electron structure, which is similar to the alkaline-earth atoms, enable us to implement new techniques (see Fig. 1). First, the absence of electron spins in the ground state is expected to suppress heating and atom loss caused by spin relaxation. Second, an ultranarrow transition of ${ }^{1} S_{0}-{ }^{3} P_{0}$ (the natural linewidth $\sim 10 \mathrm{mHz}$ [12]), which is an excellent candidate for an atomic clock $[12,13]$ and whose optical frequency was measured with an uncertainty of $4.4 \mathrm{kHz}$ [14], enables us to detect small energy differences such as the pairing gap of Fermi superfluidity. Third, the metastable ${ }^{3} P_{2}$ state has a large magnetic dipole moment of $3 \mu_{B}$ ( $\mu_{B}$ is the Bohr magneton), resulting in a factor of 9 larger magnetic dipole-dipole interaction than in the case of alkali atoms, and thus studies of anisotropic superfluidity can be expected. The large mass is also an important feature of $\mathrm{Yb}$ atoms, which leads to possibilities for interesting heteronuclear molecules with large mass difference [15]. These unique properties make production of a degenerate Fermi gas of $\mathrm{Yb}$ atoms quite appealing.

In this Letter, we report all-optical formation of degenerate fermionic ${ }^{173} \mathrm{Yb}$ gases. First, laser-cooled atoms are transferred from a magneto-optical trap (MOT) to a vertically and horizontally crossed far-off-resonance trap (FORT). Reducing the trap depth of the horizontal FORT, we performed evaporative cooling in a six-spin-state mixture in the optical dipole trap. At the final stage of evapo-

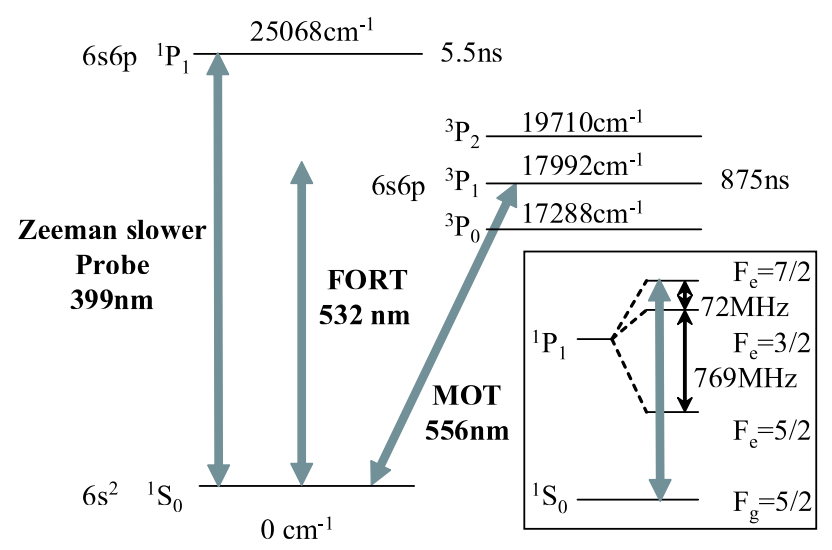

FIG. 1 (color online). Energy levels of ${ }^{173} \mathrm{Yb}$ atoms. The inset shows the hyperfine structure of the ${ }^{1} P_{1}$ state. The wavelengths of the lasers for cooling, trapping, and probing are also represented. 
ration, where the trap depth is a factor of 300 smaller than initially, the temperature of atom clouds is approximately half of the Fermi temperature, where we assume that the six magnetic substates are equally distributed. Besides the cooling of ${ }^{173} \mathrm{Yb}$ atoms, we also investigated scattering among different spin components of ${ }^{173} \mathrm{Yb}$ atoms, because the scattering length is one of the most important parameters in the study of ultracold dilute atoms.

The experiments are performed in essentially the same setup previously used to achieve BEC in ${ }^{174} \mathrm{Yb}$ [16]. First, $\mathrm{Yb}$ atoms are decelerated by a Zeeman slower with a strong transition $\left({ }^{1} S_{0}-{ }^{1} P_{1}, F_{g}=\frac{5}{2} \rightarrow F_{e}=\frac{7}{2}, 399 \mathrm{~nm}\right)$ and then cooled and collected by the MOT on an intercombination transition $\left({ }^{1} S_{0}-{ }^{3} P_{1}, F_{g}=\frac{5}{2} \rightarrow F_{e}^{\prime}=\frac{7}{2}, 556 \mathrm{~nm}\right)$ [17], as shown in Fig. 1. The MOT beam is generated by a dye laser whose frequency is narrowed to less than $100 \mathrm{kHz}$ (the linewidth of the ${ }^{1} S_{0}-{ }^{3} P_{1}$ transition $\Gamma / 2 \pi$ is $182 \mathrm{kHz}$ ) and stabilized by an ultralow expansion cavity, whose stability is typically less than $20 \mathrm{~Hz} / \mathrm{s}$. The intensity of each MOT beam is typically $60 I_{s}\left(I_{s}=0.14 \mathrm{~mW} / \mathrm{cm}^{2}\right.$ is the saturation intensity of the ${ }^{1} S_{0}-{ }^{3} P_{1}$ transition) and the detuning is $-7 \Gamma$. The loading time for the MOT is typically 30 seconds, which enables us to prepare $2 \times 10^{7}{ }^{173} \mathrm{Yb}$ atoms in the MOT.

The intensity of the MOT beam is decreased to efficiently transfer the atoms from the MOT to the FORT. In the case of ${ }^{174} \mathrm{Yb}$, which has no electronic and nuclear spin in the ground state, the intensity is decreased down to $5 I_{s}$ in our system, which results in a temperature for the MOT clouds of $50 \mu \mathrm{K}$. However, ${ }^{173} \mathrm{Yb}$ has nuclear spin $I=\frac{5}{2}$ and effective radiation pressure due to the MOT beam is smaller as a result of optical pumping [18]. For this reason, in this ${ }^{173} \mathrm{Yb}$ experiment, the intensity is lowered to $25 I_{s}$. The temperature of ${ }^{173} \mathrm{Yb}$ in the MOT is $15 \mu \mathrm{K}$, which is low compared with the ${ }^{174} \mathrm{Yb}$ MOT because of a subDoppler cooling mechanism $[17,19]$.

The laser-cooled atoms are transferred to the crossed FORT which consists of horizontal and vertical beams. The beams are independently produced by two $10 \mathrm{~W}$ diodepumped solid-state lasers at $532 \mathrm{~nm}$ and the $1 / e^{2}$ beam radii at the crossing point are $15 \mu \mathrm{m}$ (horizontal beam) and $24 \mu \mathrm{m}$ (vertical beam). The initial trap depths are $620 \mu \mathrm{K}$ (horizontal beam, full power of $5.7 \mathrm{~W}$ ) and $30 \mu \mathrm{K}$ (vertical beam, $700 \mathrm{~mW}$ ), and thus $2 \times 10^{6}$ atoms at $100 \mu \mathrm{K}$ are trapped mainly in the horizontal FORT, whose radial and axial trapping frequencies at full power are $3.6 \mathrm{kHz}$ and $30 \mathrm{~Hz}$, respectively. The radial trapping frequency is measured by parametric resonance methods [20]. In this Letter, the Fermi temperature of trapped atoms is defined for each magnetic substate. Here we assume that six spin components are equally distributed because ${ }^{173} \mathrm{Yb}$ atoms in the ground state have no electron spins and the effect of a magnetic field is small. Therefore the Fermi temperature for each spin state is the same and approximately $5 \mu \mathrm{K}$ at this stage.

We measure atom numbers and temperatures using the absorption imaging technique. The trapping beams are turned off within a few hundred ns and at a time-of-flight (TOF) time later the released gas is illuminated by a linearly polarized probe beam pulse, resonant with the ${ }^{1} S_{0}-{ }^{1} P_{1}\left(F_{g}=5 / 2 \rightarrow F_{e}=7 / 2\right)$ transition, propagating along the direction of a $0.9-\mathrm{G}$ magnetic field. The intensity of the beam is $0.02 I_{s, 1 P 1}$, where $I_{s, 1 P 1}$ is the saturation intensity of the ${ }^{1} S_{0}-{ }^{1} P_{1}$ transition, and the pulse duration is $100 \mu \mathrm{s}$, which is much longer than the absorption cycle of $550 \mathrm{~ns}$ at this intensity. It should be noted that transition rates are different for each spin component and thus the total absorption rate generally depends on the distribution of the spin components. However, it turned out from numerical calculations based on the rate equation that, in our case, the total absorption rate is the same within $2 \%$ for any initial spin distribution because of the rapid redistribution due to the optical pumping, which enables us to determine the total number of atoms exactly, without knowing the population of each component.

To perform efficient evaporation, a large elastic collision rate is necessary. At low temperature, the elastic collisions are well described by a few partial waves because, when temperatures are much less than the threshold energy $E_{\mathrm{th}}(l)$ for a given partial wave $l$ (and there is not a shape resonance [21-23]), the collisions associated with the partial wave higher than $l$ are negligible. This threshold energy can be approximately determined by the centrifugal barrier and the $C_{6} / R^{6}$ van der Waals potential:

$$
E_{\mathrm{th}}(l)=2\left(\frac{\hbar^{2} l(l+1)}{6 \mu}\right)^{3 / 2} C_{6}^{-1 / 2},
$$

where $\mu$ is the reduced mass. In the case of fermionic atoms, we can consider only the $s$-wave scattering between nonidentical fermions at temperatures much less than the $p$-wave threshold energy $E_{\mathrm{th}}(l=1)$ because there is no $s$-wave scattering between identical fermions. Therefore, the $s$-wave scattering length between nonidentical atoms plays an important role in the final stage of evaporation toward degenerate Fermi gases.

We carried out the cross-dimensional rethermalization technique to deduce the scattering length [24]. Assuming the van der Waals coefficient $C_{6}=2000$ a.u. [25], the $p$-wave threshold energy is $43 \mu \mathrm{K}$. Therefore we measure rethermalization at $6 \mu \mathrm{K}$, where the $s$-wave scattering is dominant unless there exists a $p$-wave shape resonance. Typically $1.2 \times 10^{4}$ atoms at $6 \mu \mathrm{K}$ are prepared in the crossed FORT and then a $40 \%$ amplitude modulation of $800 \mathrm{~Hz}$, twice the trapping frequency of one horizontal direction, is applied to the power of the vertical FORT during $5 \mathrm{~ms}$, which causes heating along the horizontal direction. Following the modulation, thermal relaxation is observed as the time evolution of temperatures in the horizontal and vertical directions (Fig. 2). An exponential fit to the data extracts a rethermalization time $\tau=4 \pm$ $1 \mathrm{~ms}$. To confirm this relaxation is not due to anharmonic mixing, we have also applied this technique with the same configuration of the FORT for ${ }^{176} \mathrm{Yb}$ atoms, whose scat- 


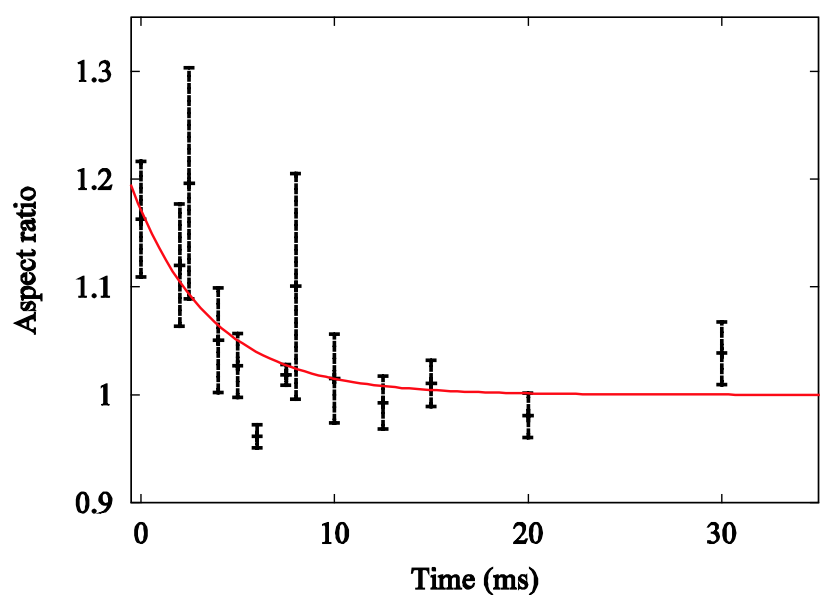

FIG. 2 (color online). Rethermalization process after anisotropic heating of the atom cloud. We caused heating along the horizontal direction and observed rethermalization as the time evolution of temperatures in the horizontal and vertical directions. An exponential fit to the data is shown by the solid line. The rethermalization time $\tau$ is $4 \mathrm{~ms}$.

tering length is not large [26]. The result for the ${ }^{176} \mathrm{Yb}$ atoms is that the rethermalization time is observed to be longer than $50 \mathrm{~ms}$, which means that the relaxation due to the anharmonicity is not less than $50 \mathrm{~ms}$; therefore, the anharmonicity effect is negligible. In the case of ${ }^{173} \mathrm{Yb}$ atoms, if we assume the atoms are equally populated among the six spin states, the rethermalization time $\tau$ and the elastic collision cross section $\sigma$ are related by $\frac{\alpha}{\tau}=$ $\frac{5}{6} \bar{n} \sigma \bar{v}$, where $\bar{n}=\int n^{2}(\vec{r}) d^{3} r / \int n(\vec{r}) d^{3} r$ is the average density and $\bar{v}=4 \sqrt{\left(k_{B} T\right) /(\pi m)}$ is the mean relative velocity with $k_{B}$ the Boltzmann constant and $m$ the ytterbium mass. The factor $\frac{5}{6}$ comes from the fact that there are no $s$-wave collisions between identical spin components. The constant $\alpha$ represents how many elastic collisions lead to cross-dimensional rethermalization and turned out to be about 2.7 by the Monte Carlo simulation [24]. From this analysis, we have deduced the absolute value of the scattering length $\left|a_{s}\right|=6 \pm 2 \mathrm{~nm}$, whose error is mainly caused by the uncertainty in the time $\tau$ and the density $\bar{n}$. It is noted that the scattering length would be larger than this value if six spin components are not equally distributed. This large scattering length allows us to cool the atoms to the quantum degenerate regime.

Forced evaporative cooling is carried out by ramping down the intensity of the horizontal beam while the vertical FORT power is constant. In $2 \mathrm{~s}$, the potential depth of the horizontal FORT is reduced to $30 \mu \mathrm{K}$, which is the same as the one of the vertical FORT, and typically $8 \times 10^{4}$ atoms are trapped in the crossed region. The temperature is about $4 T_{F}$, where the Fermi temperature $T_{F}$ is $1.4 \mu \mathrm{K}$ at this reduced FORT power. Further decrease of the horizontal FORT power results in the evaporation in the crossed region, and the potential depth along the vertical direction, which is considerably affected by gravity at the final stage of evaporation, is reduced below $2 \mu \mathrm{K}$. A few thousand atoms remain in the trap, whose mean trapping frequency is $450 \mathrm{~Hz}$, and the temperature is approximately half of $T_{F}$ $(T \simeq 180 \mathrm{nK})$.

Quantum degeneracy is observed as a decrease in cooling efficiency [1]. Figure 3 shows the number of atoms $N$ and the temperature renormalized by the Fermi temperature $\bar{T}=T / T_{F}$ at each stage of the evaporation. A line represents a trajectory of evaporation with the cooling efficiency $\gamma=3 \frac{d(\ln T)}{d(\ln N)}=2.4$, which is extracted from a fit to the data above the Fermi temperature. Below the Fermi temperature, the efficiency falls due to Fermi pressure and Pauli blocking [1].

In order to produce more degenerate gases, we have increased the volume of the optical trap by changing the beam waist and the power of the vertical beam to $86 \mu \mathrm{m}$ and $4.7 \mathrm{~W}$. The evaporation scheme is basically the same as described above, that is, lowering the intensity of the horizontal beam while that of the vertical beam is kept constant. Owing to this change, we can obtain approximately a factor of 5 more atoms at half the Fermi temperature, which enables us to do further cooling. After $4.5 \mathrm{~s}$ of evaporation, $1 \times 10^{4}$ atoms are cooled down to $\sim 75 \mathrm{nK}$, corresponding to $T / T_{F}=0.37 \pm 0.06$. The error comes from an uncertainty of $\pm 8 \%$ in the temperature and $\pm 6 \%$ in the trapping frequency. The temperature is determined by a Fermi-Dirac fit to the data (Fig. 4) [27]. We also extract $T / T_{F}=0.36 \pm 0.04$ from the fugacity [27] and these values are consistent.

In conclusion, we have succeeded in cooling fermionic ${ }^{173} \mathrm{Yb}$ atoms down to $0.37 T_{F}$, where quantum degeneracy is observed as the decrease in the evaporation efficiency. We have also deduced that the absolute value of the scattering length is approximately $6 \mathrm{~nm}$.

It is worth noting that, in this experiment, we can produce degenerate Fermi gases without preparing a two-

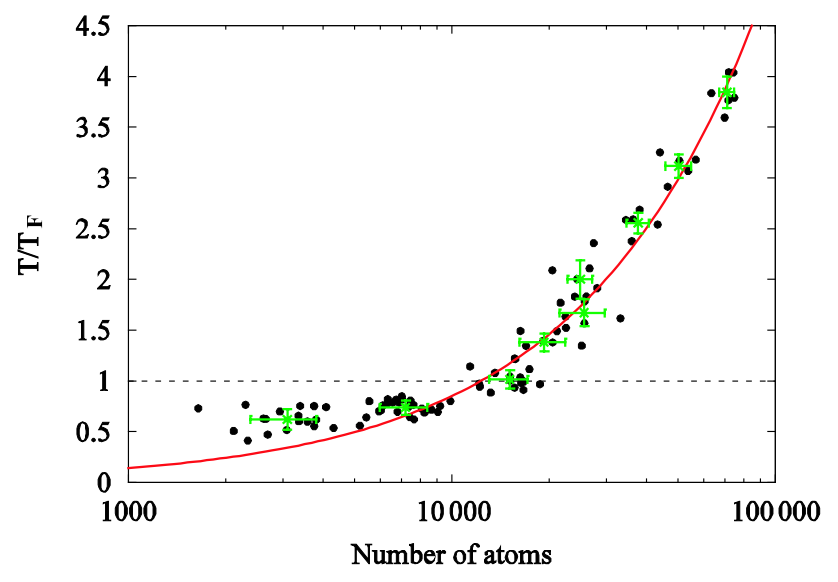

FIG. 3 (color online). Trajectory of evaporative cooling in the crossed FORT. The data of evaporation are shown as the solid circles. The solid line shows the evaporation trajectory with $\gamma=$ 2.4. Although the evaporation efficiency is nearly constant above the Fermi temperature $T_{F}$, it decreases below $T_{F}$ because of Fermi pressure and Pauli blocking. 


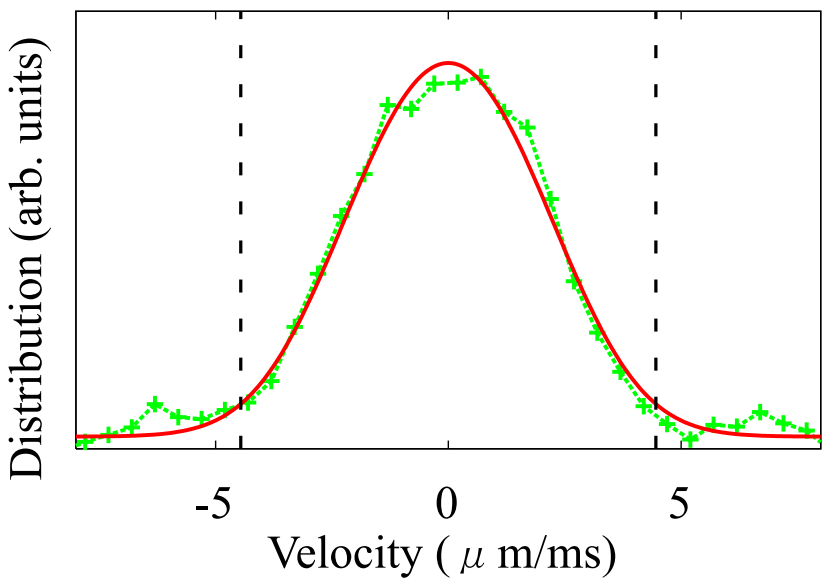

FIG. 4 (color online). Velocity distribution after $4.5 \mathrm{~s}$ of evaporation. The data are averaged over five measurements. The solid line shows a Fermi-Dirac distribution with $T / T_{F}=0.37$. The two dashed lines correspond to the Fermi velocity $v_{F}=$ $4.4 \mu \mathrm{m} / \mathrm{ms}$.

spin mixture which is stable against spin relaxation. From this result, we deduce that ${ }^{173} \mathrm{Yb}$ is less affected by spin relaxation because it has no electron spin. Recently, physics of a more-than-two component Fermi gas is attracting theoretical interests [28]. However, to our knowledge, no stable three (or more) component degenerate Fermi gas has been realized. Degenerate gases of the $\mathrm{Yb}$ experiment may have potential for this new physics.

We acknowledge T. Yabuzaki for helpful discussion, and we thank all members of $\mathrm{Yb}$ experiment group at Kyoto. This research was partially supported by Grant-in-Aid for Scientific Research of JSPS (No. 18043013, No. 18204035), SCOPE-S, and 21st Century COE "Center for Diversity and Universality in Physics" from MEXT of Japan. Y. Takasu would like to acknowledge support from JSPS.

[1] B. DeMarco and D. S. Jin, Science 285, 1703 (1999).

[2] G. Roati, F. Riboli, G. Modugno, and M. Inguscio, Phys. Rev. Lett. 89, 150403 (2002).

[3] A. G. Truscott, K. E. Strecker, W. I. McAlexander, G. B. Partridge, and R. G. Hulet, Science 291, 2570 (2001).

[4] F. Schreck, L. Khaykovich, K. L. Corwin, G. Ferrari, T. Bourdel, J. Cubizolles, and C. Salomon, Phys. Rev. Lett. 87, 080403 (2001).

[5] Z. Hadzibabic, C. A. Stan, K. Dieckmann, S. Gupta, M. W. Zwierlein, A. Görlitz, and W. Ketterle, Phys. Rev. Lett. 88, 160401 (2002).

[6] C. Silber, S. Günther, C. Marzok, B. Deh, P. W. Courteille, and C. Zimmermann, Phys. Rev. Lett. 95, 170408 (2005).
[7] S.R. Granade, M.E. Gehm, K.M. O'Hara, and J.E. Thomas, Phys. Rev. Lett. 88, 120405 (2002).

[8] C. A. Regal, M. Greiner, and D. S. Jin, Phys. Rev. Lett. 92 , 040403 (2004); M. Bartenstein et al., ibid. 92, 120401 (2004); M. W. Zwierlein et al., ibid. 92, 120403 (2004); J. Kinast et al., ibid. 92, 150402 (2004); T. Bourdel et al., ibid. 93, 050401 (2004); G. B. Partridge et al., ibid. 95, 020404 (2005).

[9] G. Roati, E. de Mirandes, F. Ferlaino, H. Ott, G. Modugno, and M. Inguscio, Phys. Rev. Lett. 92, 230402 (2004).

[10] M. Köhl, H. Moritz, T. Stöferle, K. Günter, and T. Esslinger, Phys. Rev. Lett. 94, 080403 (2005).

[11] J. M. McNamara, T. Jeltes, A. S. Tychkov, W. Hogervorst, and W. Vassen, Phys. Rev. Lett. 97, 080404 (2006).

[12] S. G. Porsev and A. Derevianko, Phys. Rev. A 69, 042506 (2004).

[13] M. Takamoto, F.-L. Hong, R. Higashi, and H. Katori, Nature (London) 435, 321 (2005).

[14] C. W. Hoyt, Z. W. Barber, C. W. Oates, T. M. Fortier, S. A. Diddams, and L. Hollberg, Phys. Rev. Lett. 95, 083003 (2005).

[15] D. S. Petrov, C. Salomon, and G. V. Shlyapnikov, J. Phys. B 38, S645 (2005).

[16] Y. Takasu, K. Maki, K. Komori, T. Takano, K. Honda, M. Kumakura, T. Yabuzaki, and Y. Takahashi, Phys. Rev. Lett. 91, 040404 (2003).

[17] T. Kuwamoto, K. Honda, Y. Takahashi, and T. Yabuzaki, Phys. Rev. A 60, R745 (1999).

[18] Y. Takasu, K. Honda, K. Komori, T. Kuwamoto, M. Kumakura, Y. Takahashi, and T. Yabuzaki, Phys. Rev. Lett. 90, 023003 (2003).

[19] R. Maruyama, R. H. Wynar, M. V. Romalis, A. Andalkar, M. D. Swallows, C. E. Pearson, and E. N. Fortson, Phys. Rev. A 68, 011403(R) (2003).

[20] S. Friebel, C. D'Andrea, J. Walz, M. Weitz, and T. W. Hänsch, Phys. Rev. A 57, R20 (1998).

[21] H. M. J. M. Boesten, C. C. Tsai, B. J. Verhaar, and D. J. Heinzen, Phys. Rev. Lett. 77, 5194 (1996).

[22] J.P. Burke, Jr., C. H. Greene, J. L. Bohn, H. Wang, P. L. Gould, and W. C. Stwalley, Phys. Rev. A 60, 4417 (1999).

[23] S. Tojo, M. Kitagawa, K. Enomoto, Y. Kato, Y. Takasu, M. Kumakura, and Y. Takahashi, Phys. Rev. Lett. 96, 153201 (2006).

[24] C. R. Monroe, E. A. Cornell, C. A. Sackett, C. J. Myatt, and C.E. Wieman, Phys. Rev. Lett. 70, 414 (1993).

[25] From recent photoassociation spectroscopy experiment, the value of $C_{6}$ is estimated to be approximately 2000 a.u. K. Enomoto et al. (to be published).

[26] Y. Takasu, T. Fukuhara, M. Kitagawa, M. Kumakura, and Y. Takahashi, Laser Phys. 16, 713 (2006).

[27] B. DeMarco, Ph.D. thesis, University of Colorado, Boulder, 2001.

[28] C. Honerkamp and W. Hofstetter, Phys. Rev. Lett. 92, 170403 (2004); P. F. Bedaque and J. P. D'Incao, cond-mat/ 0602525; T. Paananen, J.-P. Martikainen, and P. Törmä, Phys. Rev. A 73, 053606 (2006). 